\title{
Ultra high-energy neutrino at GZK energy: Z-WW showering in dark halo and tau airshowers emerging from the Earth
}

\author{
Fargion D. ${ }^{a b}$ \\ ${ }^{a}$, Rome University La Sapienza, Ple.A.Moro,2, Rome, Italy

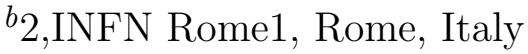

\begin{abstract}
Relic neutrino $\nu_{r}$ light masses clustering in Galactic and Local Hot Dark Halos act as a beam dump calorimeter. Ultra High Energy $\nu$, above ZeV, born by AGNs,GRBs at cosmic edges, overcoming the Greisen, Zatsepin, Kuzmin (GZK) cut-off, may hit near Z resonance and WW-ZZ channels energies: their showering into nucleons and $\gamma$ Ultra High Cosmic Ray (UHECR) fit observed data. Any tiny neutrino mass splitting may reflect into ( $a$ twin ) bump at highest GZK energy cut-off. The lighter the neutrino masses the higher the Z-Showering cut-off. The Z or WW,ZZ showering might explain a peculiar clustering in observed UHECR spectra at $10^{19}, 2 \cdot 10^{19}$, $4 \cdot 10^{19} \mathrm{eV}$ found recently by AGASA. Coincidence of clustered UHECR with highest $\gamma$ BLac sources, originated either by neutral and charged particles $(\mathrm{Q}=0,+1,-1)$ is well tuned to Z-Showering Scenario. Additional prompt TeVs signals occur offering a natural solution of growing Infrared-TeV cut-off paradoxes related to distant TeV BLac sources, while electromagnetic cascades tail may explain correlation found with GeV-EGRET Sources. Such UHE $\nu$ Astrophysics might trace near GZK energy into Horizontal Tau Air-Showers originated by the UHE $\nu_{\tau}$ Earth-Skimming in wide Crown Earth Crust around the observer. These Upward and Horizontal $\tau$ Air-Showers UPTAUS, HORTAUS, test huge crown target volumes either from high mountains as well as observing from planes, balloons or satellites. The HORTAUS from mountains measure crown masses at UHE $\nu$ EeVs energies comparable to few $\mathrm{km}^{3}$, while from satellites at orbit altitudes, at GZK energies $E_{\nu} \geq 10^{19}$, their corresponding Horizontal Crown Masses may even exceed $150 \mathrm{~km}^{3}$. The expected event rate may produce at least a dozen of event a year within Z-WW Showering model from a satellite altitude.
\end{abstract}

Keywords: Ultra High Cosmic Ray,GZK,Neutrino Tau, Tau Air Showers

\section{INTRODUCTION}

Light Neutrinos $(\sim 0.1-3 \mathrm{eV})$ clustering in Galactic, Local Hot Dark Halo, being an efficient calorimeter for $\mathrm{ZeV} \nu$ offer the possibility to overcome the Cosmic Black Body opacity ( $24 \cdot 10^{19} \mathrm{eV}$ ) (GZK) at highest energy cosmic ray astrophysics. These rare events, being nearly isotropic, are probably of cosmic origin. They are very possibly originated by blazars Jets AGN sources pointing their huge linear accelerators to us, as BLac; in standard scenario if the UHECR are originally of hadronic nature they must be absorbed by the dragging friction of cosmic $2.75 \mathrm{~K} \mathrm{BBR}$ or by the inter-galactic radio backgrounds (the GZK cut-off). Indeed as it has been noted (K.Greisen, ${ }^{26}$ Zat'sepin, Kuz'min ${ }^{38}$ 1966), proton and nucleons mean free path at $\mathrm{E}$ $>5 \cdot 10^{19} \mathrm{EeV}$ is less than $30 \mathrm{Mpc}$ and asymptotically nearly ten $\mathrm{Mpc}$; also gamma rays at those energies have even shorter interaction length $(10 \mathrm{Mpc})$ due to severe opacity by electron pair production via microwave and radio background interactions (J.W.Elbert, P.Sommers, ${ }^{8}$ 1995)(R.J.Protheroe and Biermann, ${ }^{33} 1997$. Nevertheless these powerful sources (AGN, Quasars, GRBs) suspected to be the unique source able to eject such UHECRs, are rare at nearby distances $(\lesssim 10 \div 20 M p c$, as for nearby $M 87$ in Virgo cluster); moreover there are not nearby $A G N$ in the observed UHECR arrival direction cone. Strong and coherent galactic ${ }^{33}$ or extra-galactic magnetic fields, ${ }^{9}$ able to bend such UHECR (proton, nuclei) directions, are not really at hand.

Further author information: (Send correspondence to Daniele Fargion)

E-mail: daniele.fargion@roma1.infn.it, Telephone: +390649914287

Address: Physics Department, Rome University 1,Ple.A.Moro 2,00185, Rome, Italy 
The needed coherent lengths and strength are not easily compatible with known cosmic magnetic fields. ${ }^{8}$ Finally in this scenario the $Z e V$ neutrons born, by photo-pion proton conversions on BBR, may escape the magnetic fields bending and should keep memory of the arrival direction, leading to (unobserved) clustering toward the primary source (Fargion et all 2001a, ${ }^{12}{ }^{13} 2001 \mathrm{~b}$ ). Secondaries EeV photons (by neutral pion decays) should also abundantly point and cluster toward the same nearby $A G N$ sources (P.Bhattacharjee et all $\left.^{5} 2000\right)$,(J.W.Elbert, P.Sommers, ${ }^{8}$ 1995), in disagreement with $A G A S A$ data (for any direct UHECR. Therefore Galactic origin for UHECR might be imagined as a simplest solution (by Micro-Quasars sources), but it contradicts the absence of any evident quadruple (galactic plane) or dipole (galactic halo) UHECR an-isotropy. A often revived solution of the present GZK puzzle, the Topological defects $(T D)$, assumes as a source, relic heavy particles (GUT masses) of early Universe; they are imagined diffused as a Cold Dark Matter component, in galactic halo, but therefore they are unable to explain the growing evidences of clustering in AGASA UHECR arrival data and their self-correlation with far Compact Blazars (BLac) at cosmic distance (Tinyakov P.G.et Tkachev ${ }^{35} 2001$; D.S.Gorbunov, P.G.Tinyakov, I.I.Tkachev, S.V.Troitsky ${ }^{25}$ 2002). In this frame work it is important to remind the Fly's Eye event (300 EeV) whose association with Seyfert Galaxy MCG 8-11-11 (or Quasar 3C147), inspired earliest articles $\left({ }^{11}\right.$ D.Fargion, B.Mele, A.Salis $\left.{ }^{15} 1997-99\right)$ to solve GZK by Z-Shower. Therefore the solution of UHECR puzzle based on primary Extreme High Energy (EHE) neutrino beams (from AGN) at ZeV $E_{\nu}>10^{21}$ $\mathrm{eV}$ and their undisturbed propagation from cosmic distances up to nearby calorimeter, made by relic light $\nu$ in dark galactic or local dark halo $\left({ }^{11}\right.$ D.Fargion, B.Mele, A.Salis ${ }^{15} 1997-99$,Weiler ${ }^{36}$ 1999, S.Yoshida, G. Sigl, S. Lee $\left.{ }^{37} 1998\right)$ remains the most favorite convincing solution for the GZK puzzle. New complex scenarios for each neutrino mass spectra are then opening and important signatures of $\mathrm{Z}, \mathrm{WW}$ showering must manifest in observed an-isotropy, composition, spectra shape and space-time clustering of present and future UHECR data.

\subsection{UHE $\nu$ Astronomy Energy Windows}

Rarest TeVs gamma signals are at present the most extreme and rarest trace of High Energy Astrophysics. The TeVs signals have shown new power-full Jets blazing to us from Galactic or extragalactic edges. At PeVs energies astrophysical Gamma cosmic rays should also be present, but, excluded a very rare and elusive Cyg X3 event, they are not longer being observed. While the corresponding PeVs charged cosmic rays are abundantly hitting the atmosphere, these missing PeVs gamma sources are very probably mostly absorbed by their photon interactions (photo-pion productions, electron pairs creation) at the source environment and/or along the photon propagation into the cosmic Black Body Radiation (BBR) or into other diffused (radio,infrared,optical) background radiation. Unfortunately $\mathrm{PeVs}$ charged cosmic rays, bend and bounded in a random walk by Galactic magnetic fields, loose their original directionality and their astronomical relevance; their resident time in the galaxy is much longer $\left(\geq 10^{3}-10^{5}\right)$ than neutral ones, as gamma rays, making the charged cosmic rays more probable to be observed by nearly a comparable ratio. On the contrary astrophysical UHE neutrino signals at $10^{13} \mathrm{eV}-10^{19} \mathrm{eV}$ (or higher GZK energies) are unaffected by any radiation cosmic opacity and may open a very new exciting window to High Energy Astrophysics. Lower energy astrophysical UHE $\nu$ at $10^{9} \mathrm{eV}-10^{12} \mathrm{eV}$ should also be present, but their signals are (probably) drowned by the dominant diffused atmospheric $\nu$ secondaries noises produced by the same charged (and smeared) UHE cosmic rays (while hitting terrestrial atmosphere), the so called atmospheric neutrinos. In a very far corner, at lowest (MeVs) energy windows, the abundant and steady solar neutrino flux and the prompt (but rarer) neutrino burst from a nearby Super-Novae (SN 1987A), have been in last twenty years, already successfully explored. The UHE $10^{13} \mathrm{eV}-10^{16} \mathrm{eV} \nu$ 's astronomy, being weakly interacting and rarer, may be captured mainly inside huge volumes, bigger than Super-Kamiokande ones; at present most popular detectors consider underground ones (Cubic Kilometer Size like AMANDA-NESTOR) or (at higher energy $10^{19} \mathrm{eV}-10^{21} \mathrm{eV}$ ) the widest Terrestrial atmospheric sheet volumes (Auger-Array Telescope or EUSO atmospheric Detectors). Underground $\mathrm{km}^{3}$ detection is based mainly on $\nu_{\mu}$ (above hundred $\mathrm{TeVs}$ energies, after their interaction with matter) leading to $\mu$ kilometer size lepton tracks ${ }^{22}$. Rarest atmospheric horizontal shower are also expected by $\nu$ interactions in air (and, as we shall discuss, in the Earth Crust). While $\mathrm{km}^{3}$ detectors are optimal for PeVs neutrino muons, the Atmospheric Detectors (AUGER-EUSO like) exhibit a minimal threshold at highest $\left(\geq 10^{18} \mathrm{eV}\right)$ energies. 


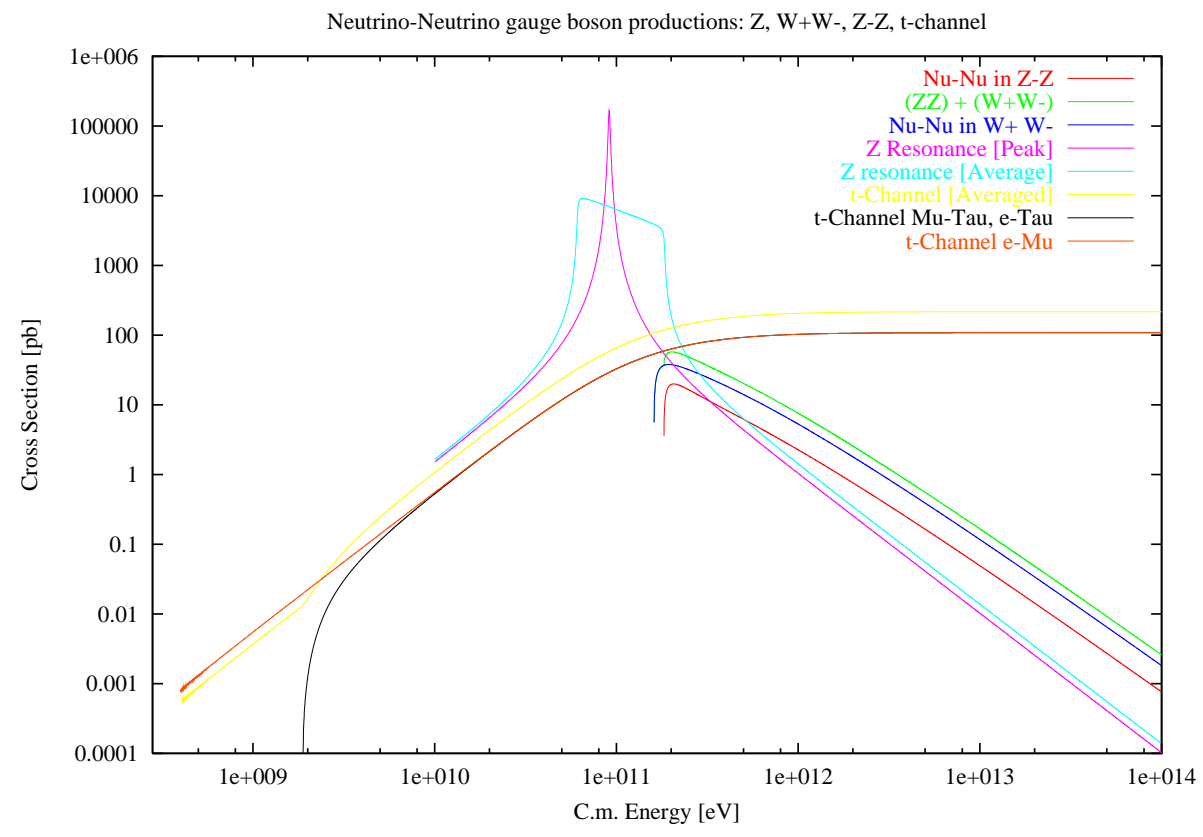

Figure 1. The $\nu \bar{\nu} \rightarrow Z, W^{+} W^{-}, Z Z, T$-channel, cross sections as a function of the center of mass energy in $\nu \nu$. These cross-sections are estimated also in average $(Z)$ as well for each possible t-channel lepton pairs. The averaged t-channel averaged the multiplicity of flavours pairs $\nu_{i}, \bar{\nu}_{j}$ respect to neutrino pair annihilations into Z neutral boson. The Z-WWZZ Showering has to be boosted by Lorentz transform to show their behaviour at laboratory system.

\section{RELIC $\nu_{R}$ NEUTRINO MASSES AND Z-WW CHANNELS AND SHOWERING}

If relic neutrinos have a mass much larger than their average thermal energy $\left(1.9 K^{0} \sim 5 \cdot 10^{-4} \mathrm{eV}\right)$ they may cluster in galactic or Local Group halos; at eVs masses the clustering seem very plausible and it may play a role in dark hot cosmology. ${ }^{10}$ The relevant role in astrophysics and cosmology of neutrino mass is covered in recent ${ }^{6}$ Their scattering with incoming extra-galactic EHE neutrinos create relativistic Z whose nucleon decay in cascades could contribute or dominate the observed UHECR flux at $G Z K$ edges.

Neutrino mass existence related to its flavour mixing has been reinforced by Super-Kamiokande evidence for atmospheric neutrino anomaly via $\nu_{\mu} \leftrightarrow \nu_{\tau}$ oscillations and more compelling evidence from Gallex and SNO solar neutrino mixing data. Consequently there are at least two main extreme scenario for hot dark halos: either $\nu_{\mu}, \nu_{\tau}$ are both extremely light $\left(m_{\nu_{\mu}} \sim m_{\nu_{\tau}} \sim \sqrt{(\Delta m)^{2}} \sim 0.05 \mathrm{eV}\right)$ and therefore energetic and fast running in hot dark neutrino halo shaped into wide, smeared and spread out to local group sizes, or $\nu_{\mu}, \nu_{\tau}$ may share nearly degenerated $(\sim \mathrm{eV})$ masses. Within the latter fine-tuned neutrino mass case $\left(m_{\nu} \sim 0.4 \mathrm{eV}\right)$ (see Fig,2), (recently found possibly hidden in a double beta decay signal) the Z peak $\nu \bar{\nu}_{r}$ interaction (see Fig.1) (D.Fargion,A.Salis, ${ }^{11}$ D.Fargion, B.Mele, A.Salis ${ }^{15}$ 1997-99,Weiler ${ }^{36}$ 1999, S.Yoshida, G. Sigl, S. Lee ${ }^{37}$ 1998) will be the favorite one.

In the second case (for heavier non constrained neutrino mass $\left(m_{\nu} \gtrsim 2-3 \mathrm{eV}\right)$ ) only a $\nu \bar{\nu}_{r} \rightarrow W^{+} W^{-}$ D.Fargion,A.Salis, ${ }^{11}$ D.Fargion, B.Mele, A.Salis ${ }^{15} 1997-99$, and the additional $\nu \bar{\nu}_{r} \rightarrow Z Z$ interactions, (see the cross-section in Fig.1)(Fargion et all. 2001a,b, ${ }^{12}{ }^{13}$ ) reconsidered here will be the only ones able to solve the GZK puzzle. Indeed the relic neutrino mass within HDM models in galactic halo near $m_{\nu} \sim 4 \mathrm{eV}$, corresponds to a lower and $Z$ resonant incoming energy $E_{\nu}=\left(\frac{4 e V}{\sqrt{m_{\nu}^{2}+p_{\nu}^{2}}}\right) \cdot 10^{21} \mathrm{eV}$. This resonant incoming neutrino energy is showering mainly a small energy fraction into nucleons $(p, \bar{p}, n, \bar{n})$, (see Tab.1 below), at energies $E_{p}$ quite below: $E_{p}=2.2\left(\frac{4 \mathrm{eV}}{\sqrt{m_{\nu}^{2}+p_{\nu}^{2}}}\right) \cdot 10^{19} \mathrm{eV}$. Therefore too heavy $(>1.5 \mathrm{eV})$ neutrino mass are not fit to solve GZK by Z-resonance; on the contrary WW,ZZ showering as well as t-channel showering may naturally keep open 


\begin{tabular}{|c|c|c|c|c|c|}
\hline \multicolumn{5}{|c|}{ Secondaries by $\nu \nu \rightarrow Z$ Interactions: $E_{\nu}=10^{22} \mathrm{eV}$, Fluence $F_{\nu}=2000 \mathrm{eVcm} \mathrm{cm}^{-2} \mathrm{~s}^{-1},\left(m_{\nu}=0.4 \mathrm{eV}\right)$} \\
\hline \hline & Multiplicity & Energy (\%) & $\sum E_{C M}(\mathrm{GeV})$ & Peak Energy (EeV) & $\frac{d N}{d E} E^{2}(\mathrm{eV})$ \\
\hline$p$ & 2.7 & $6 \%$ & 5.4 & $2.2 \cdot 10^{2}$ & 1.2 \\
\hline$\pi_{0}$ & 13 & $21.4 \%$ & 19.25 & $1.9 \cdot 10^{2}$ & 4.25 \\
\hline$\gamma_{\pi^{0}}$ & 26 & $21.4 \%$ & 19.25 & 95 & 4.25 \\
\hline$\pi^{ \pm}$ & 26 & $42.8 \%$ & 38.5 & $1.9 \cdot 10^{2}$ & 4.25 \\
\hline$\left(e^{+} e^{-}\right)_{\pi}$ & 26 & $12 \%$ & 11 & 50 & 2.3 \\
\hline$\left(e^{+} e^{-}\right)_{\text {prompt }}$ & 2 & $3.3 \%$ & 2.7 & $5 \cdot 10^{3}$ & 1.32 \\
\hline$\left(e^{+} e^{-}\right)_{\mu}$ & 2 & $1.1 \%$ & 0.9 & $1.6 \cdot 10^{3}$ & 0.45 \\
\hline$\left(e^{+} e^{-}\right)_{\tau}$ & 2 & $1.5 \%$ & 1.3 & $1.2 \cdot 10^{3}$ & 0.6 \\
\hline
\end{tabular}

Figure 2. Table 1A: The detailed energy percentage distribution into neutrino, protons, neutral and charged pions and consequent gamma, electron pair particles both from hadronic and leptonic $\mathrm{Z}, W W, Z Z$ channels. We calculated the elecro-magnetic contribution due to the t-channel $\nu_{i} \nu_{j}$ interactions. We used LEP data for $\mathrm{Z}$ decay and considered $\mathrm{W}$ decay roughly in the same way as $\mathrm{Z}$ one. We assumed that an average number of 37 particles is produced during a $\mathrm{Z}$ (W) hadronic decay. The number of prompt pions both charged (18) and neutral (9), in the hadronic decay is increased by 8 and 4 respectively due to the decay of $K^{0}, K^{ \pm}, \rho, \omega$, and $\eta$ particles. We assumed that the most energetic neutrinos produced in the hadronic decay mainly come from charged pion decay. So their number is roughly three times the number of $\pi$ 's. UHE photons are mainly relics of neutral pions. Most of the $\gamma$ radiation will be degraded around PeV energies by $\gamma \gamma$ pair production with cosmic $2.75 \mathrm{~K} \mathrm{BBR}$, or with cosmic radio background. The electron pairs instead, are mainly relics of charged pions and will rapidly lose energies into synchrotron radiation. The contribution of leptonic $\mathrm{Z}(\mathrm{W})$ decay is also considered and calculated in the table above and below.

the solution. In particular the overlapping of both the $\mathrm{Z}$ and the $\mathrm{WW}, \mathrm{ZZ}$ channels described in fig.1, for $m_{\nu} \simeq 2.3 \mathrm{eV}$ while solving the UHECR above GZK they must pile up (by Z-resonance peak activity) events at $5 \cdot 10^{19} \mathrm{eV}$, leading to a bump in AGASA data. There is indeed a first marginal evidence of such a UHECR bump in AGASA and Yakutsk data that may stand for this interpretation. More detailed data are needed to verify such very exciting possibility.

\subsection{Relic $\nu_{r}$ fine-tuned neutrino mass and the $\mathrm{Z}$ Showering-Knee}

Similar result regarding the fine tuned relic mass at $0.4 \mathrm{eV}$ and $2.3 \mathrm{eV}$ (however ignoring the WW ZZ and tchannels and invoking very hard UHE neutrino spectra) have been independently reported recently (Fodor, Katz, Ringwald ${ }^{21}$ ). We notice here (for the first time) and predict that the fine-tuned $\simeq 0.4 \mathrm{eV}$ mass (following atmospheric and solar mass splitting) being a nearly degenerated mass value, must induce, just above $2.2 \cdot 10^{20} \mathrm{eV}$, the UHECR spectra into a very sharp Z-Showering Knee,(or cut-off), (see Fig.3 above, UHECR p,n, pink curve); this Z-Knee Cut-off might be soon observable (or not) just beyond (HIRES,AGASA,AUGER) detection corner. The lighter the mass the higher energy is the Z-Knee cut-off. One of the weak point of Z-Shower model is related to the relic $\nu$ number density clustering that define the probability of the $\mathrm{Z}$ Showering. Indeed let us briefly remind the role of relic $\nu$ mass and their velocity spread within Fermi-Dirac maximal allowed number density: $n_{\nu_{i}}=1.9 \cdot 10^{3}\left(\frac{n_{\nu_{c o s m i c}}}{54 c m^{-3}}\right)\left(\frac{m_{i}}{0.1 \mathrm{eV}}\right)^{3}\left(\frac{v_{\nu_{i}}}{2 \cdot 10^{3} \frac{\mathrm{Km}}{\mathrm{s}}}\right)^{3}$. This formula imply that neutrino number density contrast is bounded by the cube of the inverse of neutrino mass and by the cube of the local Group velocity spread.

The $\nu$ number density problem may be faced either with heavier $\nu$ mass or (for lightest masses) with extreme hard UHE neutrino fluxes ${ }^{21}$ or finally with extra relic neutrino degenerancy. ${ }^{23}$ The first case is somehow exaggerating the incoming UHE $\nu$ flux, hidden just below known bounds by Goldstone experiment on $\mathrm{ZeV}$ neutrinos. The expected $\gamma$ pollution of such huge Z-Showering fluxes are also bounded by EGRET data; cosmic degenerancy (chemical potential) is increasing only by few unity the target density. However (Fargion et all 2001a,2001b) there may exist, well within or beyond Standard Cosmology, a relevant relic energetic neutrino $(M e v)$ injection due to stellar, Super-Novae, AGNs,BLacs,Gamma Ray Bursts, Soft Gamma Repeaters, Black Holes or mini-BH past activities at large redshift $\left(z \geq 10^{6}\right)$, presently red-shifted into a eV $\nu$ spectra, piling into 

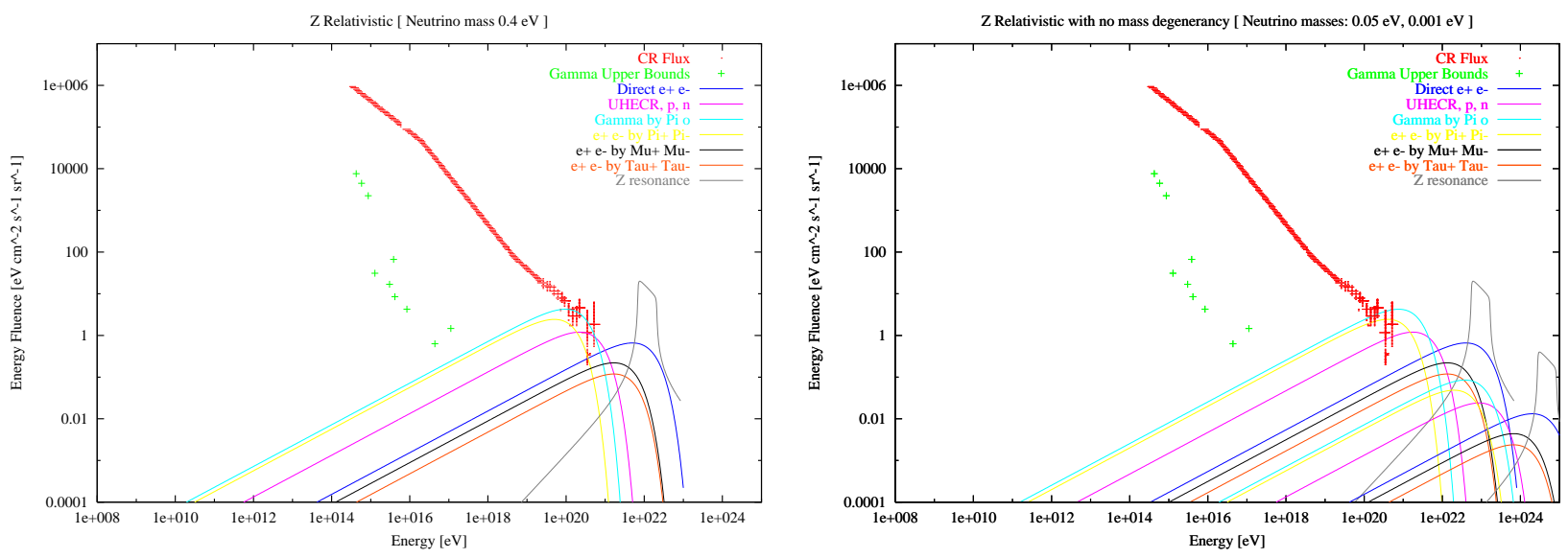

Figure 3. Left: Energy Fluence derived by $\nu \bar{\nu} \rightarrow Z$ and its showering into different channels: direct electron pairs UHECR nucleons $n p$ and anti-nucleons, $\gamma$ by $\pi^{0}$ decay, electron pair by $\pi^{+} \pi^{-}$decay, electron pairs by direct muon and tau decays as labeled in figure. The relic neutrino mass has been assumed to be fine tuned to explain GZK UHECR tail: $m_{\nu}=0.4 \mathrm{eV}$. The $\mathrm{Z}$ resonance ghost (the shadows of $\mathrm{Z}$ Showering resonance (Fargion 2001) curve), derived from $\mathrm{Z}$ cross-section in Fig.1, shows the averaged $Z$ resonant cross-section peaked at $E_{\nu}=10^{22} \mathrm{eV}$. Each channel shower has been normalized following previous table.

Figure 4. Right: Energy Fluence derived by $\nu \bar{\nu} \rightarrow Z$ and its showering into different channels as above. In the present extreme case the relic neutrino masses have been assumed with wide mass differences just compatible both with SuperKamiokande and relic $2 K^{\circ}$ Temperature. The their values have been fine tuned to explain observed GZK- UHECR tail: $m_{\nu_{1}}=0.05 \mathrm{eV}$ and $m_{\nu_{2}}=0.001 \mathrm{eV}$. A neutrino density difference between the two masses has been assumed,considering the lightest $m_{\nu_{2}}=0.001 \mathrm{eV}$ neutrino at relativistic regime. The incoming UHE neutrino fluence has been assumed growing linearly ${ }^{37}$ with energy. Its value is increased by a factor 2 and 20 at $E_{\nu_{1}}=8 \cdot 10^{22} \mathrm{eV}$ and $E_{\nu_{2}}=4 \cdot 10^{24} \mathrm{eV}$ respect the previous ones Fig.2. The "Z resonance" curve shows its averaged $Z$ resonant "ghost" cross-section peaked at $E_{\nu_{1}}=2 \cdot 10^{23} \mathrm{eV}$ and $E_{\nu_{2}}=4 \cdot 10^{24} \mathrm{eV}$, just near Grand Unification energies. Each channel shower has been normalized in analogy to table 1 .

a dense relativistic relic neutrino grey-body spectra. In this windy relativistic (or ultra relativistic) neutrino cosmology,(eventually leading to a neutrino radiation dominated Universe), the halo size (to be considered) is nearly coincident with the GZK one for UHECR nucleons and gammas $(20 \mathrm{Mpcs})$. Therefore, while the isotropic UHECR behaviour is guaranteed, a puzzle related to the non observed uniform spectra distribution seem to persist. Nevertheless the same UHE neutrino-relic neutrino scattering cross-sections (figure 1) do not follow a flat spectra, (as well as any convolutions with hypothetical $\nu$ grey body spectra). This may explain why we may leave in a homogeneous relic relativistic neutrino component at eVs energies as well as why we do observe a non-uniform UHECR spectra (reflecting non-homogeneous Z-WW-ZZ channels).

Important and often underestimated signal will produce UHE electrons pairs( by Z decay) whose final trace are TeVs photons able to break the IR-TeV cosmic cut-off. The hadronic tail of the $\mathrm{Z}$ or $W^{+} W^{-}$cascade maybe the source of final nucleons $p, \bar{p}, n, \bar{n}$ able to explain UHECR events. The same $\nu \bar{\nu}_{r}$ interactions are source of $\mathrm{Z}$ and $\mathrm{W}$ that decay in rich shower ramification ${ }^{13}$

Gamma photons at energies $E_{\gamma} \simeq 10^{20}-10^{19} \mathrm{eV}$, secondary of Z-Showering cascades, may freely propagate through galactic or local halo scales (hundreds of kpc to few Mpc) and could also contribute to the extreme edges of cosmic ray spectrum and clustering ( see: ${ }^{37},{ }^{12}{ }^{13}$ ). The ratio of the final energy flux of nucleons near the $\mathrm{Z}$ peak resonance, $\Phi_{p}$ over the corresponding electro-magnetic energy flux $\Phi_{e m}$ ratio is, as in tab.1 $e^{+} e^{-}, \gamma$ entrance, nearly $\sim \frac{1}{8}$. Moreover if one considers at higher $E_{\nu}$ energies, the opening of WW, ZZ channels and the six pairs $\nu_{e} \overline{\nu_{\mu}}, \quad \nu_{\mu} \overline{\nu_{\tau}}, \quad \nu_{e} \overline{\nu_{\tau}}$ (and their anti-particle pairs) t-channel interactions leading to highest energy leptons, with no nucleonic relics (as $p, \bar{p}$ ), this additional injection favors the electro-magnetic flux $\Phi_{e m}$ over the corresponding nuclear one $\Phi_{p}$ by a factor $\sim 1.6$ leading to $\frac{\Phi_{p}}{\Phi_{e m}} \sim \frac{1}{13}$. This ratio is valid at $W W, Z Z$ 
masses because the overall cross section variability is energy dependent. At center of mass energies above these values, the $\frac{\Phi_{p}}{\Phi_{e m}}$ decreases more because the dominant role of t-channel (Fig1). We focus here on Z, and WW,ZZ channels showering in hadrons for GZK events. The important role of UHE electron showering into $\mathrm{TeV}$ radiation is discussed below.

The minimal neutrino mass (near $0.1 \mathrm{eV}$ ) comparable with present Super-Kamiokande atmospheric neutrino mass splitting are leading to an exciting scenario where (more than one) non degenerated Z-resonances occur (Fargion et all.2001a, $\mathrm{b}^{1213}$ ). These scenario are summarized in (Fig. 2, in Ref. ${ }^{12}$ and Ref. ${ }^{13}$ ), (for nominal example $m_{\nu_{\tau}}=0.1 \mathrm{eV} ; m_{\nu_{\mu}}=0.05 \mathrm{eV}$ ). The twin neutrino mass inject a corresponding twin bump at highest energy. Another limiting case of interest takes place when the light neutrino masses are both extreme, nearly at atmospheric $(\mathrm{SK}, \mathrm{K} 2 \mathrm{~K})$ and solar (SNO) neutrino masses. This case is described in following Fig.3( keeping care of the lower number density for lightest neutrino mass). The relic neutrino masses are assumed $m_{\nu_{\tau}}=0.05$ $\mathrm{eV} ; m_{\nu_{\mu}}=0.001 \mathrm{eV}$.

The neutrino mass play a role also in defining its Hot Dark Halo size and our peculiar position in such HDM halo. Indeed for a heavy $\geq 2 \mathrm{eV}$ mass case HDM neutrino halo are mainly galactic and/or local, reflecting an isotropic or a diffused amplification toward nearby M31 HDM halo. In the lighter case the HDM should include the Local Cluster up to Virgo . To each size corresponds also a different role of UHECR arrival time. The larger the HDM size the longer the UHECR random-walk travel time and the longer the lag between doublets or triplets. The UHE neutrons by Z-Showering fits naturally the harder spectra observed in clustered events in AGASA ( ${ }^{34}$ 2001). The same UHECR neutrons may explain the quite short (2-3 years) ${ }^{34}$ lapse of time observed in AGASA doublets. The same role of UHE neutron secondaries from Z showering in HDM halo may also solve an emerging puzzle: the correlations of arrival directions of UHECRs found recently (Glushkov et all. ${ }^{27}$ 2001) in Yakutsk data at energy $E=8 \cdot 10^{18} \mathrm{eV}$ toward the Super Galactic Plane are to be compared with the compelling evidence of UHECRs events $\left(E=3 \cdot 10^{19} \mathrm{eV}\right.$ above GZK) clustering toward well defined BL Lacs at cosmic distances (redshift $z>0.1-0.2$ ) (Tinyakov et Tkachev ${ }^{35}$ 2001; Gorbunov et al. ${ }^{25}$ 2002). The question arise: where is the real UHECR sources location? At Super-galactic disk (50 Mpcs wide, within GZK range) or at cosmic ( $\geq 300 \mathrm{Mpcs}$ ) edges? Of course both results (or just one of them) maybe a statistical fluctuation. But both studies seem statistically significant (4.6-5 sigma) and they seem in some obvious disagreement. There may be a possibility for two new categories of UHECR sources. But it seem quite unnatural any propagation of direct nucleons because the UHECR from most distant BLac sources are the harder. However our Z-Showering scenario offer a common simultaneous solution: (1) The Relic Neutrino Masses define different Hierarchical Dark Halos and arrival direction correlated to Hot (Anisotropic) Relic Neutrino Halos. The real sources are at (isotropic) cosmic edges ${ }^{25}$ but their crossing along a wider anisotropic relic neutrino cloud enhance the interaction probability in the Super Galactic Plane. ${ }^{25}$ (2) The nearest SG sources are weaker while the collimated BL Lacs are harder: anyway both sources need a Neutrino Halo to induce the Z-Showering UHECRs.

\subsection{The TeV Tails from UHE Z-WW-ZZ Showering into secondary electrons}

As it is shown in Table 1 and Figures above, the electron (positron) energies by $\pi^{ \pm}$decays is around $E_{e} \sim$ $2 \cdot 10^{19} \mathrm{eV}$ for an initial $E_{Z} \sim 10^{22} \mathrm{eV}$ ( and $E_{\nu} \sim 10^{22} \mathrm{eV}$ ). Such electron pairs while not radiating efficiently in low extra-galactic magnetic fields they will be interacting with the galactic magnetic field $\left(B_{G} \simeq 10^{-6} G\right)$ leading to direct TeV photons: $E_{\gamma}^{s y n c} \sim \gamma^{2}\left(\frac{e B}{2 \pi m_{e}}\right) \sim 27.2\left(\frac{E_{e}}{2 \cdot 10^{19} \mathrm{eV}}\right)^{2}\left(\frac{m_{\nu}}{0.4 \mathrm{eV}}\right)^{-2}\left(\frac{B}{\mu G}\right) \mathrm{TeV}$. The same UHE electrons will radiate less efficiently with extra- galactic magnetic field $\left(B_{G} \simeq 10^{-9} G\right)$ leading also to direct peak $27.2 \mathrm{GeV}$ photons. The spectrum of these photons is characterized by a power of law $d N / d E d T \sim E^{-(\alpha+1) / 2} \sim E^{-1.25}$ where $\alpha$ is the power law of the electron spectrum, and it is showed in Figures above. As regards the prompt electrons at higher energy $\left(E_{e} \simeq 10^{21} \mathrm{eV}\right)$, in particular in the t-channels, their interactions with the extragalactic field first and galactic magnetic fields later is source of another kind of synchrotron emission around tens of PeV energies (Fargion et all ${ }^{13}$ 2001).

Gammas at $10^{16} \div 10^{17} \mathrm{eV}$ scatters onto low-energy photons from isotropic cosmic background $(\gamma+B B R \rightarrow$ $e^{+} e^{-}$) converting their energy in electron pair. Because the corresponding attenuation length due to the interactions with the microwave background is around ten $\mathrm{kpc}$, the extension of the halo plays a fundamental role in order to make this mechanism efficient or not. As is shown in figures above the contribution to tens of 

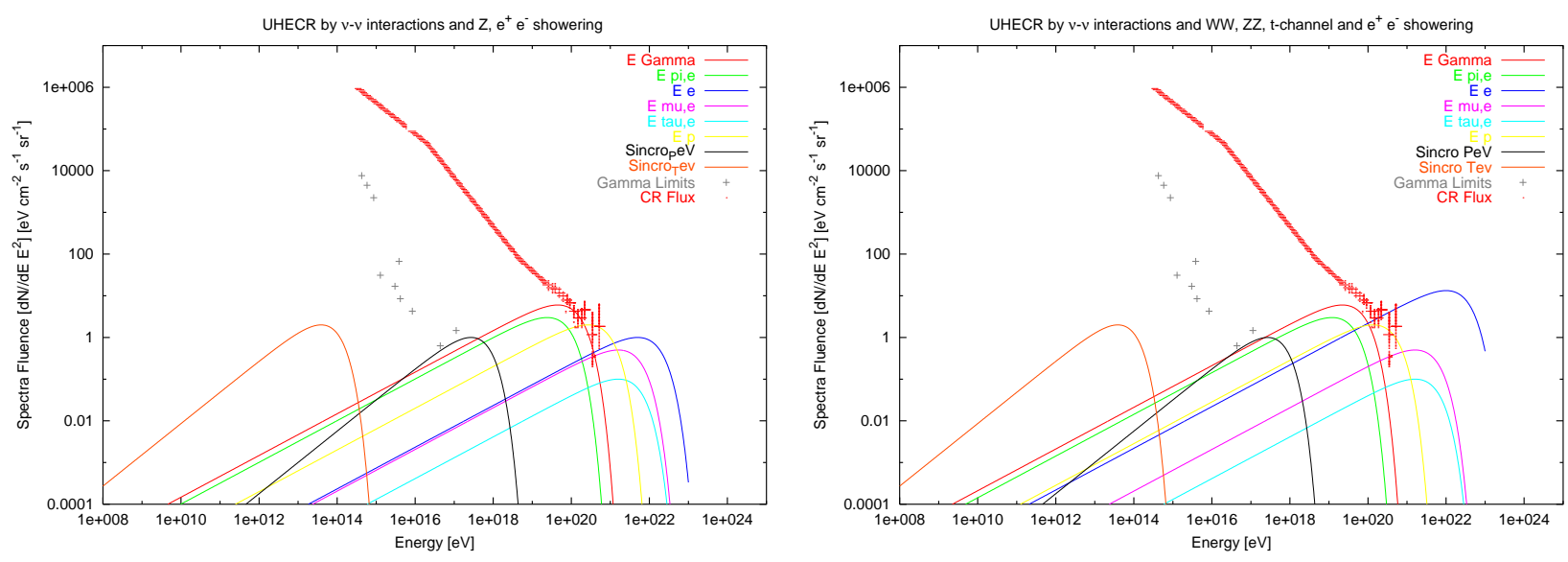

Figure 5. Left:Energy fluence by $\mathrm{Z}$ showering as above for $m_{\nu}=0.4 \mathrm{eV}$ and $E_{\nu}=10^{22} \mathrm{eV}$ and the consequent $e^{+} e^{-}$ synchrotron radiation

Figure 6. Right: Energy fluence by WW, ZZ, t-channel showering as in fig.1, for $m_{\nu}=0.4 \mathrm{eV}$ and $E_{\nu}=2 \cdot 10^{22} \mathrm{eV}$, and the consequent $e^{+} e^{-}$synchrotron radiation. The lower energy $\mathrm{Z}$ showering is not included to make spectra more understandable

PeV gamma signals by Z (or W) hadronic decay, could be compatible with actual experimental limits fixed by CASA-MIA detector on such a range of energies. Considering a halo extension $l_{\text {halo }} \gtrsim 100 \mathrm{kpc}$, the secondary electron pair creation becomes efficient, leading to a suppression of the tens of $\mathrm{PeV}$ signal. So electrons at $E_{e} \sim 3.5 \cdot 10^{16} \mathrm{eV}$ loose again energy through additional synchrotron radiation with maximum $E_{\gamma}^{s y n c}$ around $\sim 79\left(\frac{E_{e}}{10^{21} \mathrm{eV}}\right)^{4}\left(\frac{m_{\nu}}{0.4 \mathrm{eV}}\right)^{-4}\left(\frac{B}{\mu G}\right)^{3} \mathrm{MeV}$. Anyway this signal is not able to pollute sensibly the MeV-GeV while its relevance is striking by a pile up signal at TeVs. In this frame let us remind that Gamma rays with energies up to $20 \mathrm{TeV}$ have been observed by terrestrial detector only by nearby sources like Mrk $501(\mathrm{z}=0.033)$ or very recently by $\mathrm{MrK} 421(\mathrm{z}=0.031)$. More recent evidences of tens TeVs from (three times more) distant blazar 1ES1426+428 ( $\mathrm{z}=0.129)$ make even more dramatic the IR-TeV cut-off. This is puzzling because the extra-galactic $\mathrm{TeV}$ spectrum should be, in principle, significantly suppressed by the $\gamma$-rays interactions with the extra-galactic Infrared background, leading to electron pair production and TeVs cut-off. The recent calibration and determination of the infrared background by DIRBE and FIRAS on COBE have inferred severe constrains on $\mathrm{TeV}$ propagation. Indeed, as noticed by Kifune ( $\left.{ }^{30} 1997\right)$, and Protheroe and Meyer ${ }^{32}$ we may face a severe infrared background - TeV gamma ray crisis. This crisis imply a distance cut-off, incidentally, comparable to the GZK one. So our present Z-Showering scenario $\left({ }^{13}\right)$ may easily solve also the IR-TeV cut off. Let us remind also an additional evidence for IR-TeV cut-off is related to the possible discover of tens of $\mathrm{TeV}$ counterparts of BATSE GRB970417, observed by Milagrito(R. Atkins et all ${ }^{2}$ 2000), being most GRBs very possibly at cosmic edges, at distances well above the IR-TeV cut-off ones. To solve the IR-TeV cut-off one may alternatively invoke unbelievable extreme hard intrinsic spectra or exotic explanation as gamma ray superposition of photons or finally sacrilegious Lorentz invariance violation.

To conclude the puzzle one finally needs to scrutiny the UHE $\nu$ astronomy and to test the GZK solution within Z-Showering Models by any independent search on Earth for such UHE neutrinos traces above PeVs reaching either EeVs-ZeVs extreme energies.

\subsection{UHE $\nu$ Astronomy by $\tau$ Air-Shower}

Recently Fargion et al.1999,,${ }^{16}$ Fargion $2002^{18}$ proposed a new competitive UHE $\nu$ detection based on ultra high energy $\nu_{\tau}$ interaction in matter and its consequent secondary $\tau$ decay in flight while escaping from the rock (Mountain Chains) or water (Sea) in air leading to Upward or Horizontal $\tau$ Air-Showers (UPTAUs and HORTAUs),Fargion 2001a, ${ }^{17}$ Fargion $2001 b,{ }^{14}$ In a pictorial way one may compare the UPTAUs and HORTAUs as 
the double bang processes expected in $\mathrm{km}^{3}$ ice-water volumes Learned Pakvasa 1995, ${ }^{31}$ : the double bang is due first to the UHE $\nu_{\tau}$ interaction in matter and secondly by its consequent $\tau$ decay in flight. Here we consider a (hidden) UHE $\nu$-N Bang in (the rock-water within a mountain or the Earth Crust) and a $\tau$ bang out in air, whose shower is better observable at high altitudes. The main power of the UPTAUs and HORTAUs detection is the huge amplification of the UHE neutrino signal, which may deliver almost all its energy in numerous secondaries traces (Cherenkov lights, gamma, $\mathrm{X}$ photons, electron pairs, collimated muon bundles). Indeed the multiplicity in $\tau$ Air-showers secondary particles, $N_{o p t} \simeq 10^{12}\left(E_{\tau} / \mathrm{PeV}\right), N_{\gamma}\left(<E_{\gamma}>\sim 10 \mathrm{MeV}\right) \simeq 10^{8}\left(E_{\tau} / \mathrm{PeV}\right)$, $N_{e^{-} e^{+}} \simeq 2 \cdot 10^{7}\left(E_{\tau} / \mathrm{PeV}\right), N_{\mu} \simeq 3 \cdot 10^{5}\left(E_{\tau} / \mathrm{PeV}\right)^{0.85}$ makes easy the UPTAUs-HORTAUs discover. These HORTAUs, also named Skimming neutrinos,${ }^{20}$ maybe also originated on front of mountain chains $,{ }^{16},{ }^{18}{ }^{29}$ either by $\nu_{\tau} N, \bar{\nu}_{\tau} N$ interactions as well as by $\bar{\nu}_{e} e \rightarrow W^{-} \rightarrow \bar{\nu}_{\tau} \tau$. This new UHE $\nu_{\tau}$ detection is mainly based on the oscillated UHE neutrino $\nu_{\tau}$ originated by more common astrophysical $\nu_{\mu}$, secondaries of pion-muon decay at PeVs-EeVs-GZK energies. These oscillations are guaranteed by Super Kamiokande evidences for flavour mixing within GeVs atmospheric neutrino data as well as by most solid and recent evidences of complete solar neutrino mixing observed by SNO detector. HORTAUs from mountain chains must nevertheless occur, even for no flavour mixing, as being inevitable $\bar{\nu}_{e}$ secondaries of common pion-muon decay chains $\left(\pi^{-} \rightarrow \mu^{-}+\bar{\nu}_{\mu} \rightarrow e^{-}+\bar{\nu}_{e}\right)$ near the astrophysical sources at Pevs energies. They are mostly absorbed by the Earth and are only rarely arising as UPTAUS. Their Glashow resonant interaction allow them to be observed as HORTAUs only within a very narrow and nearby crown edges at horizons (not to be discussed here). At wider energies windows $\left(10^{14} \mathrm{eV}-10^{20} \mathrm{eV}\right.$ ) only neutrino $\nu_{\tau}, \bar{\nu}_{\tau}$ play a key role in UPTAUS and HORTAUS. These Showers might be easily detectable looking downward the Earth's surface from mountains, planes, balloons or satellites observer. Here the Earth itself acts as a "big mountain" or a wide beam dump target. The present upward $\tau$ at horizons should not be confused with an independent and well known, complementary (but rarer) Horizontal Tau Airshower originated inside the same terrestrial atmosphere: we shall referee to it as the Atmospheric Horizontal Tau Air-Shower. The same UPTAUS have a less competitive upward showering due to $\nu_{e} \bar{\nu}_{e}$ interactions with atmosphere, showering in thin upward air layers ${ }^{4}$ : let us label this atmospheric Upward Tau as A-UPTAUs and consider its presence as a very small additional contribute, because rock is more than 3000 times denser than air (see the ratio in last column in final Table). Therefore at different heights we need to estimate the UPTAUS and HORTAUs event rate occurring along the thin terrestrial crust below the observer, keeping care of their correlated variables.

\section{THE UPTAUS-HORTAUS SKIN EARTH CROWNS}

The $\tau$ airshowers are observable at different height $h_{1}$ leading to different underneath observable terrestrial areas and crust volumes. HORTAUs in deep valley are also relate to the peculiar geographical morphology and composition, ${ }^{18}$ as discussed below. ${ }^{19}$ We remind in this case the very important role of UHE $\bar{\nu}_{e} e \rightarrow W^{-} \rightarrow \bar{\nu}_{\tau} \tau^{-}$ channels which may be well observable even in absence of any $\nu_{\tau}, \bar{\nu}_{\tau}$ UHE sources or any neutrino flavour mixing: its Glashow peak resonance make these neutrinos unable to cross all the Earth across but it may be observable beyond mountain chain ${ }^{18}$; while testing $\tau$ air-showers beyond a mountain chain one must keep in mind the possible amplification of the signal because of a possible New TeV Physics (see Fig 9). ${ }^{18}$ In the following we shall consider in general the main $\nu_{\tau}-N, \bar{\nu}_{\tau}-N$ nuclear interaction on Earth crust. It should be kept in mind also that UPTAUs and in particular HORTAUS are showering at very low densities and their geometrical opening angle (here assumed at $\theta \sim 1^{\circ}$ ) is not in general conical (like down-ward showers) but they are more in a thin fan-like shape (like the observed 8 shaped horizontal Air-Showers). The fan shape is opened by the Terrestrial magnetic field bending. These UPTAUs-HORTAUs duration time is also much longer than common down-ward showers because their showering occurs at much lower air density: from micro (UPTAUS from mountains) to millisecond (UPTAUs and HORTAUs from satellites) long flashes. Indeed the GRO observed Terrestrial Gamma Flashes, possibly correlated with the UPTAUs ${ }^{18}$ show the millisecond duration times. In order to estimate the rate and the fluence for of UPTAUs and HORTAUs one has to estimate the observable mass, facing a complex chain of questions, leading for each height $h_{1}$, to an effective observable surface and volume from where UPTAUs and HORTAUs might be originated. ${ }^{19}$ From this effective volume it is easy to estimate the observable rates, assuming a given incoming UHE $\nu$ flux model for galactic or extragalactic sources. Here we shall only refer to the Masses estimate,unrelated to any UHE $\nu$ flux models. These steps 

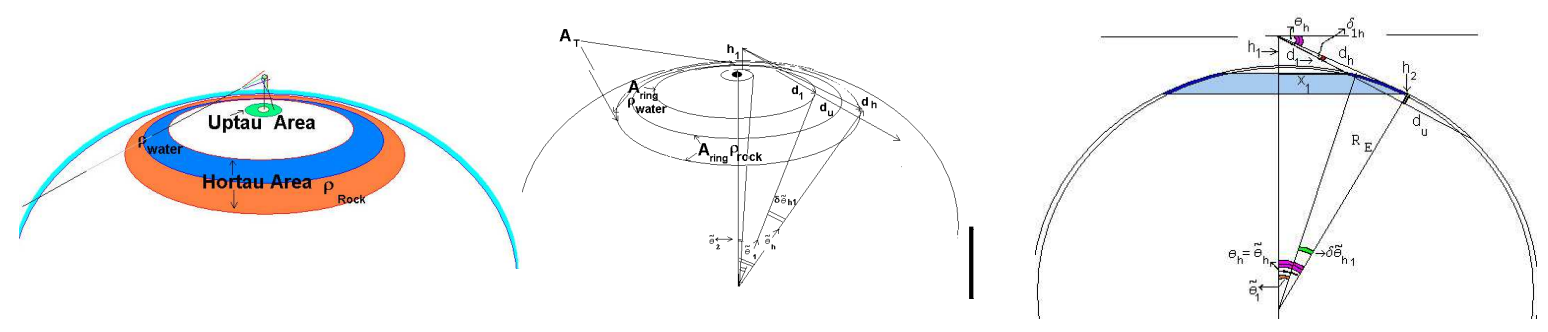

Figure 7. Left:The Upward Tau Air-Shower, UPTAUs,and the Horizontal Tau Air-Shower, HORTAU, flashing toward an observer at height $h_{1}$. The HORTAU areas are described for water and rock matter density.

Figure 8. Center: As previous figure, The Upward Tau Air-Shower, UPTAUs, HORTAU, flashing toward an observer at height $h_{1}$ from the Skin Crown Earth Crust seen in $3 D$. The HORTAU areas are described for water and rock matter density. ${ }^{19}$

Figure 9. Right:The lateral geometrical disposal of the main parameters in the text defining the UPTAUs and HORTAUs Areas; the distances are exaggerated for simplicity.

are linking simple terrestrial spherical geometry and its different geological composition, high energy neutrino physics and UHE $\tau$ interactions, the same UHE $\tau$ decay in flight and its air-showering physics at different quota within terrestrial air density. ${ }^{19}$ Detector physics threshold and background noises, signal rates have been kept in mind, ${ }^{18}$ but they will be discussed and explained in forthcoming papers. Let us therefore define, list and estimate below the sequence of the key variables whose dependence (shown below or derived in Appendices) leads to the desired HORTAUs volumes (useful to estimate the UHE $\nu$ prediction rates) summirized in a last Table and in Conclusions. Let us now show the main functions whose interdependence with the observer altitude lead to estimate the UPTAUs and HORTAUs equivalent detection Surfaces, Volumes and Masses. ${ }^{19}$ The horizontal distance $d_{h}$ toward the horizons: $d_{h}=113 \sqrt{\frac{h_{1}}{k m}} \cdot \sqrt{1+\frac{h_{1}}{2 R_{\oplus}}} k m$. The corresponding horizontal edge angle $\theta_{h}: \theta_{h}=\arccos \frac{R_{\oplus}}{\left(R_{\oplus}+h_{1}\right)} \simeq 1^{o} \sqrt{\frac{h_{1}}{k m}}$. (Approximations here and below hold for height $h_{1} \ll R_{\oplus}$.) The consequent characteristic lepton $\tau$ energy $E_{\tau_{h}}$ making decay $\tau$ in flight from $d_{h}$ distance just nearby the source: $E_{\tau_{h}}=\left(\frac{d_{h}}{c \tau_{0}}\right) m_{\tau} c^{2} \simeq 2.2 \cdot 10^{18} \mathrm{eV} \sqrt{\frac{h_{1}}{k m}} \sqrt{1+\frac{h_{1}}{2 R}}$. At low quota $\left(h_{1} \leq\right.$ a few kms) the air depth before the Tau decay necessary to develop a shower correspond to a Shower distance $d_{S h} \sim 6 \mathrm{kms} \ll d_{h}$. More precisely at low quota $\left.\left(h_{1} \ll h_{o}: h_{0}=8.55 \mathrm{~km}\right): d_{S h} \simeq 5.96 \mathrm{~km}\left[1+\ln \frac{E_{\tau}}{10^{18} \mathrm{eV}}\right)\right] \cdot e^{\frac{h_{1}}{h_{o}}}$. So we may neglect the distance of the final shower respect to the longest horizons ones. However at high altitude $\left(h_{1} \geq h_{o}\right)$ this is no longer the case . Therefore we shall introduce from here and in next steps a small, but important modification , whose physical motivation is just to include the air dilution role at highest quota: $h_{1} \rightarrow \frac{h_{1}}{1+h_{1} / H_{o}}$, where, as in Appendix A, $H_{o}=23 \mathrm{~km}$. Therefore previous definition becomes: $E_{\tau_{h}} \simeq 2.2 \cdot 10^{18} \mathrm{eV} \sqrt{\frac{h_{1}}{1+h_{1} / H_{o}}} \sqrt{1+\frac{h_{1}}{2 R}}$. This procedure, applied tacitly everywhere, guarantees that there we may extend our results to those HORTAUs at altitudes where the residual air density must exhibit a sufficient slant depth. For instance, highest $\gg 10^{19} \mathrm{eV}$ HORTAUs will be not easily observable because their $\tau$ life distance exceed (usually) the horizons air depth lenghts. The parental UHE $\nu_{\tau}, \bar{\nu}_{\tau}$ or $\bar{\nu}_{e}$ energies $E_{\nu_{\tau}}$ able to produce such UHE $E_{\tau}$ in matter: $E_{\nu_{\tau}} \simeq 1.2 E_{\tau_{h}} \simeq$ $2.64 \cdot 10^{18} \mathrm{eV} \cdot \sqrt{\frac{h_{1}}{k m}}$ The neutrino (underground) interaction lenghts at the corresponding energies is $L_{\nu_{\tau}}: L_{\nu_{\tau}}=$ $\frac{1}{\sigma_{E \nu_{\tau}} \cdot N_{A} \cdot \rho_{r}}=2.6 \cdot 10^{3} \mathrm{~km} \cdot \rho_{r}^{-1}\left(\frac{E_{\nu_{h}}}{10^{8} \cdot \mathrm{GeV}}\right)^{-0.363} \simeq 304 \mathrm{~km} \cdot\left(\frac{\rho_{\text {rock }}}{\rho_{r}}\right) \cdot\left(\frac{h_{1}}{\mathrm{~km}}\right)^{-0.1815}$. For more details see, ${ }^{22},{ }^{18} \cdot{ }^{19}$ The maximal neutrino depth $h_{2}\left(h_{1}\right)$ under the chord along the UHE neutrino-tau trajectory of lenght $L_{\nu}\left(h_{1}\right)$ : $h_{2}\left(h_{1}\right)=\frac{L_{\nu_{h}}^{2}}{2^{2} \cdot 2\left(R-h_{2}\right)} \simeq \frac{L_{\nu_{h}}^{2}}{8 R_{\oplus}} \simeq \simeq 1.81 \cdot \mathrm{km} \cdot\left(\frac{h_{1}}{k m}\right)^{-0.363} \cdot\left(\frac{\rho_{r o c k}}{\rho_{r}}\right)^{2}$ See figure above for more details. Because the above $h_{2}$ depths are in general not too deep respect to the Ocean depths, we shall consider either sea (water) or rock (ground) materials as Crown matter density. The corresponding opening angle observed from height $h_{1}, \delta_{1 h}$ encompassing the underground height $h_{2}$ at horizons edge (see Fig.2) and the nearest UHE $\nu$ arrival directions $\delta_{1}$ 

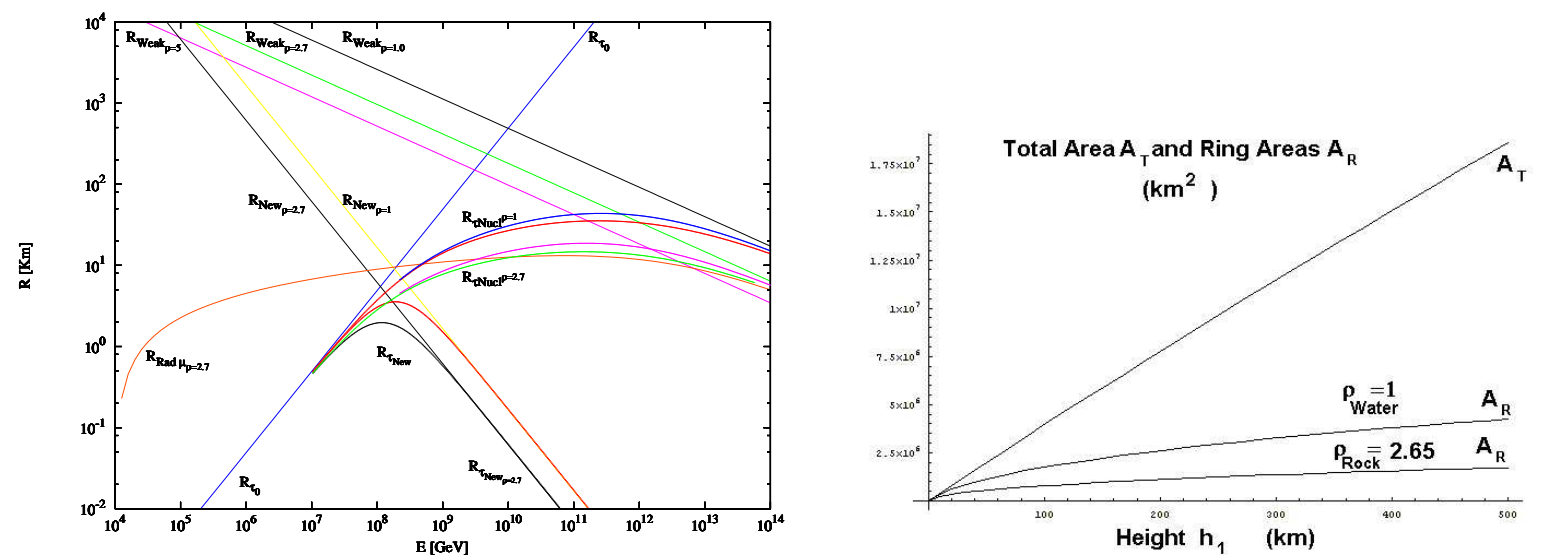

Figure 10. Left: Lepton $\tau$ (and $\mu$ ) Interaction Lenghts for different matter density: $R_{\tau_{o}}$ is the free $\tau$ lenght, $R_{\tau_{N e w}}$ is the New Physics TeV Gravity interaction range at corresponding densities, $R_{\tau_{N u c l} \cdot \rho,}{ }^{18}{ }^{18}$ see also, ${ }^{3},{ }^{7},{ }^{19}$ is the combined $\tau$ Ranges keeping care of all known interactions and lifetime and mainly the photo-nuclear one. There are two slightly different split curves (for each density) by two comparable approximations in the interaction laws. $R_{W e a k \rho}$ is the electroweak Range at corresponding densities $\left(\right.$ see also $\left.^{22}\right) ;{ }^{18} \cdot{ }^{19}$

Figure 11. Total Area $A_{T}$ and Ring Areas for two densities $A_{R}$ at high altitudes ${ }^{19}$

is $^{19}: \delta_{1 h}\left(h_{2}\right)=2 \arctan \frac{h_{2}}{2 d_{h}}=2 \arctan \left[\frac{8 \cdot 10^{-3} \cdot\left(\frac{h_{1}}{k m}\right)^{-0.863}\left(\frac{\left.\rho_{\text {rock }}\right)^{2}}{\rho_{r}}\right]}{\sqrt{1+\frac{h_{1}}{2 R}}}\right] \simeq 0.91^{\circ}\left(\frac{\rho_{\text {rock }}}{\rho_{r}}\right)^{2} \cdot\left(\frac{h_{1}}{k m}\right)^{-0.863}$ The underground chord $d_{u_{1}}$ (see Fig. $8-9$ ) where UHE $\nu_{\tau}$ propagate and the nearest distance $d_{1}$ for $\tau$ flight (from the observer toward Earth) along the same $d_{u_{1}}$ direction, within the angle $\delta_{1 h}$ defined above, angle below the horizons (within the upward UHE neutrino and HORTAUs propagation line) is ${ }^{19}: d_{u_{1}}=2 \cdot \sqrt{\sin ^{2}\left(\theta_{h}+\delta_{1 h}\right)\left(R_{\oplus}+h_{1}\right)^{2}-d_{h}{ }^{2}}$. Note that by definition and by construction: $L_{\nu} \equiv d_{u_{1}}$. The nearest HORTAUs distance corresponding to this horizontal edges still transparent to UHE $\tau$ is: $d_{1}\left(h_{1}\right)=\left(R_{\oplus}+h_{1}\right) \sin \left(\theta_{h}+\delta_{1 h}\right)-\frac{1}{2} d_{u_{1}}$ Note also that for height $h_{1} \geq k m: \frac{d_{u_{1}}}{2} \simeq\left(R_{\oplus}+h_{1}\right) \sqrt{\delta_{1 h} \sin 2 \theta_{h}} \simeq 158 \sqrt{\frac{\delta_{1 h}}{1^{\circ}}} \sqrt{\frac{h_{1}}{k m}} k m$

The same distance projected cord $x_{1}\left(h_{1}\right)$ along the horizontal line (see Fig.9): $x_{1}\left(h_{1}\right)=d_{1}\left(h_{1}\right) \cos \left(\theta_{h}+\delta_{1 h}\right)$ The total terrestrial underneath any observer at height $h_{1}$ is $A_{T}:=2 \pi R_{\oplus}^{2}\left(1-\cos \tilde{\theta}_{h}\right)=2 \pi R_{\oplus} h_{1}\left(\frac{1}{1+\frac{h_{1}}{R_{\oplus}}}\right)$; $A_{T}=4 \cdot 10^{4} \mathrm{~km}^{2}\left(\frac{h_{1}}{k m}\right)\left(\frac{1}{1+\frac{h_{1}}{R}}\right)$. Where $\tilde{\theta}_{h}$ is the opening angle from the Earth along the observer and the horizontal point whose value is the maximal observable one. At first sight one may be tempted to consider all the Area $A_{T}$ for UPTAUs and HORTAUs but because of the air opacity (HORTAUs) or for its paucity (UPTAUs) this is incorrect. While for HORTAUs there is a more complex Area estimated above and in the following, for UPTAUs the Area Ring (or Disk) is quite simpler to derive following very similar geometrical variables summirized in Appendix. The Earth ring crown crust area $A_{R}\left(h_{1}\right)$ delimited by the horizons distance $d_{h}$ and the nearest distance $d_{1}$ still transparent to UHE $\nu_{\tau}$. The ring area $A_{R}\left(h_{1}\right)$ is computed from the internal angles $\delta \tilde{\theta}_{h}$ and $\delta \tilde{\theta}_{1}$ defined at the Earth center (note that $\delta \tilde{\theta}_{h}=\delta \theta_{h}$ but in general $\delta \tilde{\theta}_{1} \neq \delta \theta_{1}$ ). $A_{R}\left(h_{1}\right)=2 \pi R_{\oplus}^{2}\left(\cos \tilde{\theta}_{1}-\cos \tilde{\theta}_{h}\right)=2 \pi R_{\oplus}^{2}\left(\sqrt{1-\left(\frac{x_{1}\left(h_{1}\right)}{R_{\oplus}}\right)^{2}}-\frac{R_{\oplus}}{R_{\oplus}+h_{1}}\right)$ Here $x_{1}\left(h_{1}\right)$ is the cord defined above. ${ }^{19}$

The characteristic interaction lepton tau lenght $l_{\tau}$ defined at the average $E_{\tau_{1}}$, from interaction in matter (rock or water). These lenghts have been derived by a analytical equations keeping care of the Tau lifetime, the photo-nuclear losses, the electro-weak losses ${ }^{18} .{ }^{19}$ See figure 10 below.

The $l_{\tau_{\downarrow}}$ projected along the $\sin \left(\delta \tilde{\theta}_{h_{1}}\right)$ is defined by: $\delta \tilde{\theta}_{h_{1}} \equiv \tilde{\theta}_{h}-\arcsin \left(\frac{x_{1}}{R_{\oplus}}\right)$ 
The same quantity in a more direct approximation: $\sin \delta \tilde{\theta}_{h_{1}} \simeq \frac{L_{\nu}}{2 R_{\oplus}}=\frac{304 k m}{2 R_{\oplus}}\left(\frac{\rho_{\text {rock }}}{\rho}\right){\frac{h_{1}}{k m}}^{-0.1815}$. From highest $\left(h \gg H_{o}=23 \mathrm{~km}\right)$ altitude the exact approximation reduces to: $\delta \tilde{\theta}_{h_{1}} \simeq 1^{o}\left(\frac{\rho_{\text {rock }}}{\rho}\right){\frac{h_{1}}{500 \cdot k m}}^{-0.1815}$. Therefore the penetrating $\tau$ skin depth $l_{\tau_{\downarrow}}$ is: $l_{\tau_{\downarrow}}=l_{\tau} \cdot \sin \delta \tilde{\theta}_{h_{1}}, \simeq 0.0462 \cdot l_{\tau}\left(\frac{\rho_{\text {water }}}{\rho}\right){\frac{h_{1}}{k m}}^{-0.1815}$. Where the $\tau$ ranges in matter, $l_{\tau}$ has been calculated and shown in Fig.10. The final analytical expression for the Earth Crust Skin Volumes and Masses under the Earth Skin inspected by HORTAUs are derived combining the above functions on HORTAUs Areas with the previous lepton Tau $l_{\tau_{\downarrow}}$ vertical depth depths: $V_{h_{1}}=A_{R}\left(h_{1}\right) \cdot l_{\tau_{\downarrow}} ; M_{h_{1}}=V_{h_{1}} \cdot\left(\frac{\rho}{\rho_{\text {water }}}\right)$. At high altitudes the above approximation corrected accordingly to the exact one, ${ }^{19}$ shown in Figure 11 , becomes: $A_{R}\left(h_{1}\right) \simeq 2 \pi R_{\oplus} d_{h 1} \delta \tilde{\theta}_{h_{1}} \simeq 4.65 \cdot 10^{6} \sqrt{\frac{h_{1}}{500 k m}}\left(\frac{\rho_{\text {water }}}{\rho}\right) k m^{2}$. The $M_{h_{1}}$ from where HORTAUs may be generated is derived as: $V_{h_{1}}=\frac{\pi}{2} \sqrt{\frac{2 h_{1}}{R_{\oplus}}}\left(\frac{\sqrt{1+\frac{h_{1}}{2 R}}}{1+\frac{h_{1}}{R}}\right) L_{\nu}^{2} l_{\tau} \propto \rho^{-3}$. Therefore $M_{h_{1}}=\frac{\pi}{2} \sqrt{\frac{2 h_{1}}{R_{\oplus}}}\left(\frac{\sqrt{1+\frac{h_{1}}{2 R}}}{1+\frac{h_{1}}{R}}\right) L_{\nu}^{2} l_{\tau} \rho \propto \rho^{-2}$. The effective observable Skin Tau Mass $\Delta M_{\text {eff. }}\left(h_{1}\right)$ within the thin HORTAU or UPTAUs Shower angle beam $\simeq$ $1^{o}$ is suppressed by the solid angle of view: $\frac{\delta \Omega}{\Omega} \simeq 2.5 \cdot 10^{-5}$. Therefore $\Delta M_{\text {eff. }}\left(h_{1}\right)=V_{h_{1}} \cdot\left(\frac{\rho}{\rho_{\text {water }}}\right) \frac{\delta \Omega}{\Omega}$. The Masses $\Delta M_{\text {eff. }}\left(h_{1}\right)$ for realistic high quota experiment are summirized in Table below, ${ }^{19}$ their consequent event rate are discussed in next section and in the Conclusion below.

\begin{tabular}{||c|c|c|c|c|c|c|c|c|c|c|c|c||}
\hline$\rho$ & $h 1$ & $E_{t h}(\mathrm{eV})$ & $A_{T O T}\left(\mathrm{~km}^{2}\right)$ & $l_{\tau}(\mathrm{km})$ & $\delta \widetilde{\theta}$ & $l_{\tau} \sin \delta \widetilde{\theta}(\mathrm{km})$ & $A_{R}\left(\mathrm{Km}^{2}\right)$ & $\frac{d \Omega}{\Omega}$ & $\Delta V(\mathrm{~km})$ & $V_{\text {eff }}=\frac{\Delta V \Delta \Omega}{\Omega}$ & $\Delta M\left(\mathrm{~km} \mathrm{~m}^{3}\right)$ & $R=\frac{M_{T}}{M_{A T M}}$ \\
\hline \hline 1 & 2 & $3.12 * 10^{18}$ & $8 * 10^{4}$ & 21.7 & $1.31^{\circ}$ & 0.496 & $7.9 * 10^{4}$ & $2.5 * 10^{-5}$ & $3.95 * 10^{4}$ & 0.987 & 0.987 & 49.6 \\
\hline 2.65 & 2 & $3.12 * 10^{18}$ & $8 * 10^{4}$ & 11 & $0.97^{\circ}$ & 0.186 & $7.2 * 10^{4}$ & $2.5 * 10^{-5}$ & $1.34 * 10^{4}$ & 0.335 & 0.89 & 49.2 \\
\hline \hline 1 & 5 & $4.67 * 10^{18}$ & $2 * 10^{5}$ & 24.3 & $1.79^{\circ}$ & 0.76 & $1.9 * 10^{5}$ & $2.5 * 10^{-5}$ & $1.45 * 10^{5}$ & 3.64 & 3.64 & 75 \\
\hline 2.65 & 5 & $4.67 * 10^{18}$ & $2 * 10^{5}$ & 12.1 & $1.07^{\circ}$ & 0.225 & $1.45 * 10^{5}$ & $2.5 * 10^{-5}$ & $3.2 * 10^{4}$ & 0.82 & 2.17 & 59.6 \\
\hline \hline 1 & 25 & $8 * 10^{18}$ & $10^{6}$ & 27.5 & $2.36^{\circ}$ & 1.13 & $7.16 * 10^{5}$ & $2.5 * 10^{-5}$ & $8.12 * 10^{5}$ & 20.3 & 20.3 & 113 \\
\hline 2.65 & 25 & $8 * 10^{18}$ & $10^{6}$ & 13.1 & $1.08^{\circ}$ & 0.247 & $3.83 * 10^{5}$ & $2.5 * 10^{-5}$ & $9.5 * 10^{4}$ & 2.4 & 6.3 & 65.45 \\
\hline \hline 1 & 500 & $1.08 * 10^{19}$ & $1.8 * 10^{7}$ & 29.4 & $2.72^{\circ}$ & 1.399 & $4.3 * 10^{6}$ & $2.5 * 10^{-5}$ & $6 * 10^{6}$ & 150.6 & 150.6 & 140 \\
\hline 2.65 & 500 & $1.08 * 10^{19}$ & $1.8 * 10^{7}$ & 13.8 & $1.07^{\circ}$ & 0.257 & $1.75 * 10^{6}$ & $2.5 * 10^{-5}$ & $4.5 * 10^{5}$ & 11.3 & 30 & 68 \\
\hline
\end{tabular}

Figure 12. The Table of the main parameters leading to the effective HORTAUs Mass from the observer height $h_{1}$, the corresponding $\tau$ energy $E_{\tau}$ able to let the $\tau$ reach him from the horizons, the Total Area $A_{T O T}$ underneath the observer, the corresponding $\tau$ propagation lenght in matter $l_{\tau}$, the opening angle toward the crown from the Earth $\delta \tilde{\theta}_{h_{1}}$ and $l_{\tau}$ just orthogonal in the matter $l_{\tau_{\downarrow}}=l_{\tau} \cdot \sin \delta \tilde{\theta}_{h_{1}}$, the Ring Areas for two densities $A_{R}$ at characteristic high altitudes $h_{1}$, the corresponding effective Volume $V_{e f f}$. and the consequent Mass $\Delta M_{e f f}$. (within the narrow $\tau$ Air-Shower solid angle) as a function of density $\rho$ and height $h_{1}$. In the last Column the Ratio $\mathrm{R}=M_{T} / M_{A T M}$ define the ratio of HORTAUs produced within the Earth Crown Skin over the atmospheric ones: this ratio nearly reflects the matter over air density and it reaches nearly two order of magnitude. ${ }^{19}$

\section{EVENT RATE FOR UPTAUS AND HORTAUS}

The event rate for HORTAUs are given by the following expression normalized to any given neutrino flux $\Phi_{\nu}$ :

$$
\dot{N}_{\text {year }}=\Delta M_{e f f} \cdot \Phi_{\nu} \cdot \dot{N}_{o} \cdot \frac{\sigma_{E_{\nu}}}{\sigma_{E_{\nu}}}
$$

Where the $\dot{N}_{o}$ is the UHE neutrino rate estimated for $\mathrm{km}^{3}$ at any given (unitary) energy $E_{\nu_{o}}$, in absence of any Earth shadow. In our case we shall normalize our estimate at $E_{\nu_{o}}=3 \mathrm{PeVs}$ energy for standard electro-weak charged current in a standard parton model ${ }^{22}$ and we shall assume a model-independent neutrino maximal flux $\Phi_{\nu}$ at a flat fluence value of nearly $\Phi_{\nu_{o}} \simeq 3 \cdot 10^{3} \mathrm{eV} \mathrm{cm}^{-2} \cdot \mathrm{s}^{-1} \cdot \mathrm{sec}^{-1} \cdot \mathrm{sr}^{-1}$ corresponding to a characteristic Fermi power law in UHE $\nu$ primary production rate decreasing as $\frac{d N_{\nu}}{d E_{\nu}} \simeq E_{\nu}{ }^{-2}$ just below present AMANDA bounds. The consequent rate becomes: $\dot{N}_{\text {year }}=29 \frac{\Delta M_{e f f}}{\mathrm{~km}^{3}} \cdot \frac{\Phi_{\nu}}{\Phi_{\nu_{o}}} \cdot \frac{\sigma_{E_{\nu}}}{\sigma_{E_{\nu_{o}}}}$

$$
\dot{N}_{\text {year }}=29 \cdot\left(\frac{E_{\nu}}{3 \cdot 10^{6} \cdot G e V}\right)^{-0.637} \frac{\Delta M_{\text {eff. }}}{k m^{3}} \cdot \frac{\Phi_{\nu}}{\Phi_{\nu_{o}}}
$$


For highest satellites and for a characteristic UHE GZK energy fluence $\Phi_{\nu_{o}} \simeq 310^{3} \mathrm{eVcm}^{-2} \cdot \mathrm{s}^{-1} \cdot \mathrm{sr}^{-1}$ (as the needed Z-Showering one), the consequent event rate observable $\dot{N}_{\text {year }}$ above the Sea is from satellite quota (500 $\mathrm{km})$ :

$$
\dot{N}_{\text {year }}=12.3 \cdot\left(\frac{E_{\nu}}{3 \cdot 10^{10} \cdot G e V}\right)^{-0.637} \cdot \frac{\Phi_{\nu}}{\Phi_{\nu_{o}}}
$$

This event rate is comparable to UPTAUS one and it may be an additional source of Terrestrial Gamma Flashes observed by GRO in last decade, ${ }^{18} \cdot{ }^{19}$

\section{SUMMARY AND CONCLUSIONS}

The discover of the expected UHE neutrino Astronomy is urgent and just behind the corner. Huge volumes are necessary. Beyond underground $\mathrm{km}^{3}$ detectors a new generation of UHE neutrino calorimeter lay on front of mountain chains and just underneath our feet: The Earth itself offers huge Crown Volumes as Beam Dump calorimeters observable via upward Tau Air Showers, UPTAUs and HORTAUs. Their effective Volumes as a function of the quota $h_{1}$ has been derived by an analytical function variables in equations above and Appendix. These Volumes and Masses are discussed below and summirized in the last column of the above Table; they are large enough to offer an ideal calorimeter for future UHE neutrino detection.

\section{APPENDIX : THE UPTAUS AREA}

The Upward Tau Air-Showers, mostly at PeV energies, might travel a minimal air depth before reaching the observer in order to amplify its signal. The UPTAUS Disk Area $A_{U}$ underneath an observer at height $h_{1}$ within a opening angle $\tilde{\theta}_{2}$ from the Earth Center is: $A_{U}=2 \pi R_{\oplus}^{2}\left(1-\cos \tilde{\theta}_{2}\right)$. Where the $\sin \tilde{\theta}_{2}=\left(x_{2} / R_{\oplus}\right)$ and $x_{2}$ behaves like $x_{1}$ defined above for HORTAUs. In general the UPTAUs area are constrained in a narrow Ring

(because the mountain presence itself or because the too near observer distances from Earth are encountering a too short air slant depth for showering or a too far and opaque atmosphere for the horizontal UPTAUs): $A_{U}=2 \pi R_{\oplus}{ }^{2}\left(\cos \tilde{\theta}_{3}-\cos \tilde{\theta}_{2}\right)$ An useful Euclidean approximation is: $A_{U}=\pi h_{1}{ }^{2}\left(\cot \theta_{2}{ }^{2}-\cot \theta_{3}{ }^{2}\right)$ Where $\theta_{2}, \theta_{3}$ are the outgoing $\tau$ angles on the Earth surface, ${ }^{18} \cdot{ }^{19}$

\section{REFERENCES}

1. T.Aoki et al. ICRC 2001, pp. 915-918.2001

2. R. Atkins et.al, astro-ph/0001111, 2000.

3. F. Becattini,S. Bottai, 2001, Astropart.Phys. 15, 323

4. V.S Berezinzky,et al., 1990, Astrophysics of Cosmic Rays (North Holand ed.)

5. P. Bhattacharjee, G.Sigl, Phys.Report. 327, pp. 109-247,2000

6. A.Dolgov in Phys.Report. 2002 (in press); hep-ph/0202122.

7. Iyer Dutta, S., Reno, M. H., Sarcevic, I. \& Seckel, D.2001,Phys.Rev. D63, 094020.

8. J.W. Elbert, Sommers, P., ApJ 441, 151,1995.

9. G.R.Farrar et all., Phys.Rev.Lett. 84, 3527, 2000

10. D.Fargion, Nuovo Cimento 77B,111,1983

11. D.Fargion,A. Salis, Proc. 25th ICRC HE 4-6, pp. 153-156.1997

12. D.Fargion, M.Grossi, P.G.De Sanctis Lucentini, C.Di Troia,R.V.Konoplich, astro-ph/0102426, Dark 2000,Heidelberg,10-14,July 2000, Ed.H.V.Klapdor-Kleingrothaus, Springer,2001a, pp. 455-468.

13. D.Fargion, M.Grossi, P.G.De Sanctis Lucentini, C.Di Troia. hep-ph/0108050 ,J.Phys.Soc.Jpn. Vol(70) Supplement B, pp. 46-57, 2001.

14. D.Fargion, 27th ICRC 2001HE1.8,Germany, pp. 903-906, astro-ph/0107094.

15. D. Fargion, B. Mele, A. Salis 1999, Ap. J. 517, 725; astro-ph/9710029.

16. D. Fargion, A. Aiello,R. Conversano, 1999, 26th ICRCHE6.1.10, pp. 396-398; astro-ph/9906450.

17. D.Fargion, 27th ICRC 2001 HE2.5, pp. 1297-1300, astro-ph/0106239;

18. D.Fargion, Ap. J.570, 909-927, 2002; astro-ph/0002453; also astro-ph/9704205; 
19. D.Fargion, hep-ph/0206010.

20. J.L. Feng, P. Fisher, F. Wilczek, M.Yu Terri ; hep-ph/0105067

21. Z. Fodor, S.D. Katz, A. Ringwald hep-ph/0105336,2001

22. R. Gandhi, C. Quigg, , M. H. Reno, I. Sarcevic, 1998, Phys. Rev. D 58, 093009

23. G. Gelmini, A. Kusenko Phys.Rev.Lett. 84 (2000)

24. Yu.A.Golubkov, R.V. Konoplich,Phys.Atom.Nucl. 61,602,1998

25. D.S.Gorbunov, P.G.Tinyakov, I.I.Tkachev, S.V.Troitsky, astro-ph/0204360, ApJL (in press 2002)

26. K.Greisen, Phys.Rev.Lett. 16,748,1966

27. A.V. Glushkov ,I.Ye.Sleptov ICRC 2001, pp. 414-416,2001

28. N. Hayashida et all.AGASA collaboration,Astropart.Phys. 10 (1999), pp. 303-311.

29. G.W.S. Hou, M.A. Huang; astro-ph/0204145.

30. T.Kifune, Astrophys.J.Lett. 518, L21.1999

31. J. G. Learned ,S. Pakvasa, 1995, Astropart. Phys. 3, 267

32. R.J.Protheroe, H.Meyer, astro-ph/0005349, 2000

33. R.J.Protheroe, P.L.Biermann, AstroPart.Phys. 7,181,1997

34. M.Takeda and AGASA Collab., J.Phys.Soc.Jpn. Vol.70, pp. 15-21,2001.

35. P. Tinyakov ,I.Tkachev , J.Phys.Soc.Jpn. Vol.70, pp. 58-63,2001

36. T.J.Weiler, Astropart.Phys. 11, pp. 303-316.1999

37. S.Yoshida, G. Sigl, S. Lee,Phys.Rev.Lett. 81, pp. 5505-5508.1998

38. G.T.Zat'sepin, V.A Kuz'min, , JETP Lett. 4,78,1966 